\title{
Tríada de Lenk como presentación clínica del angiomiolipoma
}

\author{
Pedemonte JG, Degiovanni D, Pusterla D, Reibel C, Di Nucci J, Boccio C, Degiovanni R, Dotta A. \\ Departamento de Cirugía. Servicio de Urología. Hospital Italiano Garibaldi. Instituto Universitario Italiano. \\ Rosario, Santa Fe, Argentina.
}

Actas Urol Esp. 2008;32(8):850-854

\section{RESUMEN}

TRÍADA DE LENK COMO PRESENTACIÓN CLÍNICA DEL ANGIOMIOLIPOMA

$\mathrm{El}$ angiomiolipoma (AML) es una neoplasia clonal benigna compuesta por cantidades variables de tejido adiposo maduro, músculo liso y vasos sanguíneos de paredes gruesas. Aproximadamente un $20 \%$ de todos los AML, se detectan en pacientes con el Síndrome de la Esclerosis Tuberosa, trastorno autonómico dominante que se asocia a retraso mental, epilepsia, adenoma sebáceo.

La presencia de hemorragia retroperitoneal masiva, conocida como Síndrome de Wunderlich se ha documentado en hasta un 10\% de los pacientes y representa la complicación más importante y temida de estas lesiones.

La TAC identifica las zonas del tejido adiposo, correspondiente a una baja atenuación entre 20-100 Hounsfield, dicho coeficiente dependerá del contenido y madurez de la grasa intratumoral y de la presencia o no de hemorragias.

En el caso de pacientes con AML pequeños que requieren intervención debido a la presencia de síntomas, en pacientes con ET o AML multicéntricos y en pacientes en quienes la preservación de la función renal reviste particular importancia el enfoque preferido es la cirugía ahorradora de nefronas con nefrectomía parcial o embolizaciòn selectiva.

Presentamos un caso de una paciente de 59 años con un angiomiolipoma gigante quién debuta con el Síndrome clínico conocido como Tríada de Lenk.

Palabras clave: Angiomiolipoma. Sindrome Lenk. Hemorragia retroperitoneal.

\section{ABSTRACT \\ ANGIOMYOLIPOMA AND LENK SYNDROME: CASE REPORT}

Angiomyolipoma (AML) is a benign clonal neoplasm that consists of varying amounts of mature adipose tissue, smooth muscle, and thick-walled vessels.

Approximately 20\% of AMLs are found in patients with tuberous sclerosis syndrome (TS), an autosomaldominant disorder characterized by mental retardation, epilepsy, and adenoma sebaceous, a distinctive skin lesion.

Massive retroperitoneal hemorrhage from AML, also known as Wunderlich's syndrome, has been found in up to $10 \%$ of patients and represents the most significant and feared complication.

The presence of even a small amount of fat within a renal lesion on CT scan (confirmed by Hounsfield unit's $\leq 10$ ) is considered diagnostic of AML. Intervention should be considered for larger tumors, particularly if the patient is symptomatic, taking into account patient age, comorbidities, and other related factors. A nephron-sparing approach, via either partial nephrectomy or selective embolization, is clearly preferred.

We present the case of a fifty-nine-year old patient with an angiomyolipoma and massive retroperitoneal hemorrhage with Lenk syndrome. 
$\mathrm{E}$ 1 angiomiolipoma (AML) es una neoplasia clonal benigna compuesta por cantidades variables de tejido adiposo maduro, músculo liso y vasos sanguíneos de paredes gruesas.

Se encuentra presente en un 0,3\% riñones examinados en autopsias, y entre un 2-5\% de la población evaluada por ecografía. Brissar y Bourneville fueron los primeros en describirlo en pacientes afectos de Esclerosis Tuberosa (ET) en 1880, aunque en un principio se denominó de distintas formas (mioangiolipoma, arterioleiomioma benigno), fue Morgan quién en 1951 acuñó el nombre actual ${ }^{1-3}$.

Morfológicamente son hamartomas, es decir, tumores benignos constituidos por tejidos normalmente presentes en el órgano en que asientan, pero anormales en cantidad, disposición o grado de maduración.

Microscópicamente siempre están integrados por tres componentes en proporciones variables: tejido adiposo, fibras musculares lisas y vasos neoformados. El tejido adiposo es casi siempre de tipo adulto, los vasos neoformados suelen ser de tipo arterial muscular y arteriolas, trayecto tortuoso y con anomalías estructurales en su pared, que facilitan la hemorragia; las fibras musculares lisas adquieren diversas morfologías ${ }^{3-5}$.

En la conducta terapéutica del AML se debe tener en cuenta la evolución natural y sobre todo el riesgo de hemorragia asociado con estas lesiones ${ }^{12,13}$.

Presentamos un caso clínico de una paciente con angiomiolipoma asociado a hemorragia retroperitoneal espontánea y Tríada de Lenk.

\section{CASO CLÍNICO}

Paciente de sexo femenino de 59 años que consulta a guardia médica por dolor abdominal agudo de 12 horas de evolución localizado en hipocondrio izquierdo asociados a nauseas, vómitos, astenia, mareos y sudoración profusa.

Antecedentes personales: Diabetes Mellitus en tratamiento con hipoglucemiantes orales.

Angiomiolipoma diagnosticado hace 15 años sin control posterior.

Signos vitales: FR 16/min; FC 95 1/m; TA 90/50 $\mathrm{mmHg}$.

A la exploración presenta masa palpable renitente a la palpación profunda sobre el hipocondrio asociado a signos de peritonismo (abdomen agudo).
La analítica informa: hematíes $3.81 \mathrm{Mill} / \mathrm{mm} 3$, hemoglobina $10,2 \mathrm{~g} / \mathrm{dl}$, hematocrito $22 \%$, leucocitos $18200 \mathrm{~mm} 3$, urea $54 \mathrm{mg} / \mathrm{dl}$, creatinina $0,9 \mathrm{ng} / \mathrm{ml}$, eritrosedimentación $29 \mathrm{~mm}$, glicemia $293 \mathrm{mg} \%$.

Se transfunden 5 U [GR] y 2 U de plasma fresco congelado.

Se solicita tomografía axial computada(TAC) de abdomen y pelvis con contraste y evaluación prequirúrgica.

La TAC informó: Masa ocupante de espacio que crece a partir del seno renal compuesta por tejido graso que mide 180-166-360 $\mathrm{mm}$ asociado a hematoma perirrenal que desplaza al yeyuno-íleon y colapsa al colon descendente (Figs. 1 y 2).

Se realizó nefrectomía radical izquierda de urgencia por vía anterior (subcostal izquierda).

El informe de la Anatomía patológica fue: angiomiolipoma con áreas de hemorragia que mide 19-

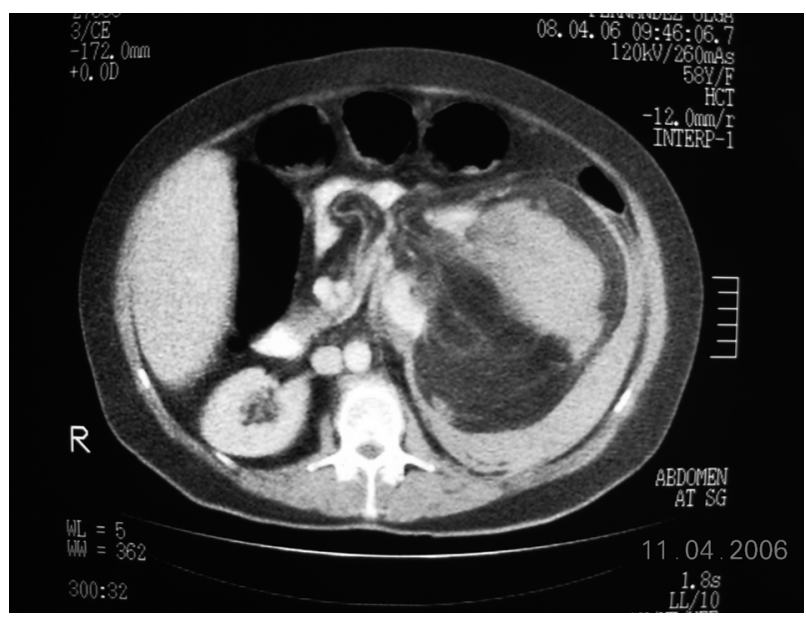

FIGURA 1

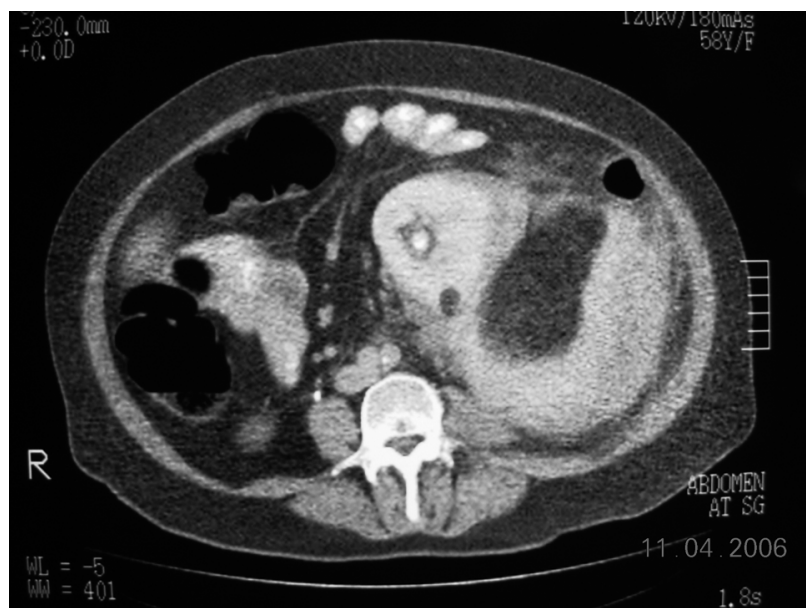

FIGURA 2 
$17.5-8 \mathrm{~cm}$ y pesa $1.500 \mathrm{~g}$. La lesión comprime la vía pielocalicial y estructuras del hilio, sin signos de infiltración. Parénquima renal asiento de cambios congestivos y mínima exudación inflamatoria crónica. No existe evidencia de desviación maligna (Figs. 3 y 4).

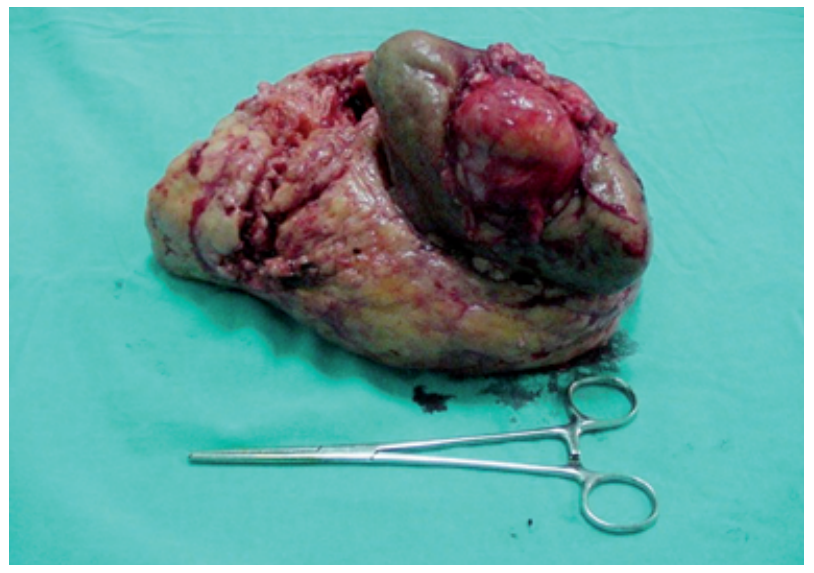

FIGURA 3

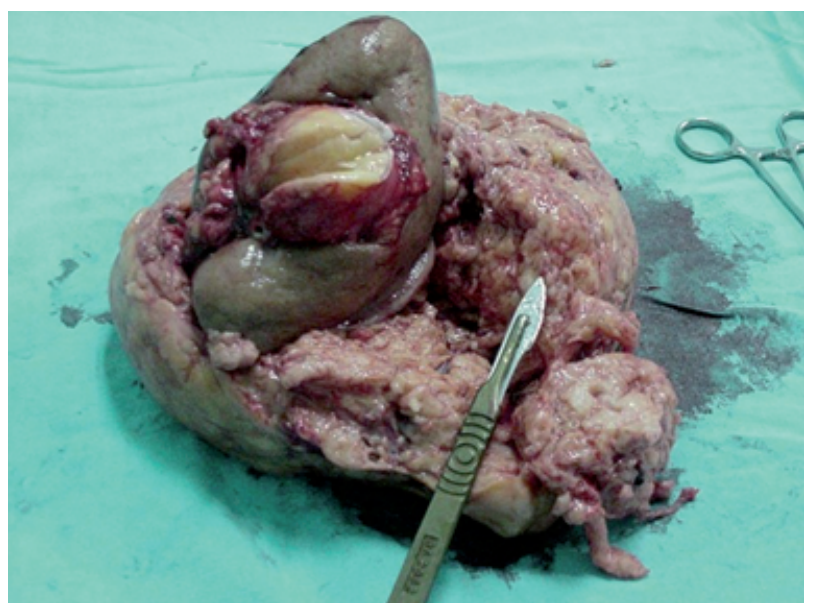

FIGURA 4

Actualmente la paciente se encuentra asintomática, reintegrada a sus tareas cotidianas.

\section{DISCUSIÓN}

Aproximadamente un $20 \%$ de todos los AML, se detectan en pacientes con el Síndrome de la Esclerosis Tuberosa, trastorno autonómico dominante que se asocia a retraso mental, epilepsia, adenoma sebáceo, lesión cutánea distintiva y alteraciones en riñón, corazón, pulmón y otros órganos, debido a la proliferación combinada de células de origen ectodérmico y mesodérmico ${ }^{1,3,6,7}$. La pene- trancia de cada uno de estos rasgos dista de ser completa y alrededor de un $50 \%$ de los pacientes con ET desarrollan AML.

En estos pacientes, suelen ser múltiples, bilaterales y afectan a personas más jóvenes con una relación mujer: hombre de 2:1.

En los pacientes con AML sin ET (80\%) su incidencia es cada vez mayor llegando a cifras de incidentaloma del $15 \% \%^{3,8}$. Suelen afectar con mayor frecuencia a mujeres $(4 / 1)$ en edades comprendidas entre 40 y 60 años, siendo una masa única y solitaria de mayor tamaño que los asociados a ET. La presentación clínica, difiere si el AML es esporádico o si se encuentra dentro del cuadro clínico de la ET.

Cuando el AML se presenta en forma aislada éste puede ser asintomático y ser diagnosticado tras una exploración abdominal asociada a otras causas; o bien presentarse dentro de un abanico de signos y sintomas tales como: dolor abdominal o en el flanco, hematuria micro o macroscópica, hemorragia retroperitoneal, hipertensión arterial, náuseas, vómitos, fiebre, anemia, infección urinaria y fallo renal ${ }^{1,3,7,10}$.

La tríada clásica más frecuente de presentación es el dolor abdominal (84\%), masa abdominal (54\%) $y$ hematuria (40\%).

La presencia de hemorragia retroperitoneal masiva se ha documentado en hasta un 10\% de los pacientes y representa la complicación más importante y temida de estas lesiones. El cuadro se presenta con la tríada de Lenk: dolor agudo, masa palpable y signo de hemorragia interna (shock hipovolémico), requiriendo tratamiento inmediato ${ }^{1,3,6,10}$. Este cuadro fue descrito por primera vez por Wunderlich en 1856 otorgándole el nombre de apoplejía espontánea de la cápsula renal, calificativo que ha sido sustituido por el de hematoma perirrenal espontáneo o Síndrome de Wunderlich ${ }^{2}$. La hemorragia es consecuencia de la vascularidad aumentada de un tejido deficiente en elasticidad o por una invasión venosa directa ${ }^{4,5}$.

La asociación entre hipertensión y AML podría explicarse por la compresión del tumor sobre el parénquima renal, que provocaría una isquemia del mismo, o la existencia de fístulas intratumorales responsables de la desviación del flujo sanguíneo renal $^{9-11}$.

El embarazo aumenta el riesgo de hemorragia secundaria a un AML, y este factor puede afectar la adopción de decisiones clínicas. 
Una cuarta parte de ellos se ubican fuera del parénquima renal, siendo entre otros el hígado, el útero y el retroperitoneo los lugares más comunes ${ }^{6-8}$.

Dada su naturaleza benigna el diagnóstico preoperatorio es un paso fundamental para planear un adecuado tratamiento.

El urograma excretor no logra diferenciar el AML del adenocarcinoma renal. En algunos casos que se presentan con sindrome de Wunderlich, el estudio mostrará una masa que ocupa la región lumbar y borra la línea del psoas con cierto grado de contractura antiálgica, elevación del hemidiafragma correspondiente y desviación de uréter lumbo-sacro hacia la línea media.

Por su contenido graso, la apariencia ecográfica es hiperecogénica (secundaria a las propiedades acústicas del tejido graso, así como del gran número de interfases titulares entre los diferentes componentes del tumor, lo que condiciona una mayor reflexión de la onda sonora, al poseer cada tejido distinta impedancia acústica).

Aunque típica, dicha apariencia no es patognomónica, pudiéndose presentar en pacientes con adenocarcinomas, linfomas, lipomas y oncocitomas.

Si bien la ecografía aporta una sospecha diagnóstica, la principal utilidad radica en ser la técnica de elección en el seguimiento de los pacientes con AML teniendo en cuenta su inocuidad, disponibilidad y bajo coste.

El estudio de elección para confirmar la presencia de un AML es la Tomografía axial computada (TAC). La TAC identifica las zonas del tejido adiposo, correspondiente a una baja atenuación entre 20100 Hounsfield, dicho coeficiente dependerá del contenido y madurez de la grasa intratumoral y de la presencia o no de hemorragias. El carcinoma renal no contiene grasa, sin embargo el liposarcoma renal y retroperitoneal deben incluirse en el diagnóstico diferencial, aunque la presencia de grasa inmadura y abundante estroma con altos valores de atenuación lo diferencian del AML ${ }^{10-12}$

Las limitaciones de la TAC dependerán de la cantidad de grasa, ya que cuando el componente vascular y muscular es alto, el diagnóstico preoperatorio es de carcinoma renal, y si predomina el componente adiposo, se sospechará un lipoma o liposarcoma.

La Resonancia Nuclear Magnética ha demostrado ser más sensible que la TAC en la identificación de la grasa (T2 largo en áreas de alta intensidad); por lo tanto será de elección en algunas casos ambiguos por otras modalidades diagnósticas. Se puede utilizar en pacientes con insuficiencia renal, aunque tiene la desventaja de ser costosa y más lenta ${ }^{10-12}$.

La arteriografía es evocadora pero no específica, no siendo útil para el diagnóstico diferencial con el carcinoma renal ${ }^{11,12}$

En casos de sangrado activo, nos permitirá localizar el sitio del mismo, permitiendo una embolizaciòn selectiva arterial o planeamiento de la cirugía renal.

En la conducta terapéutica del AML se debe tener en cuenta la evolución natural y sobre todo el riesgo de hemorragia asociado con estas lesiones. En general la mayor parte de los AML sintomáticos son relativamente grandes y en casi todos los estudios publicados se define un diámetro umbral de $4 \mathrm{~cm}^{3,9-13}$.

Los AML multicéntricos y aquellos que se presentan en pacientes con ET representan un grupo especial de tumores con velocidad de crecimiento aumentada (20\% por año frente a un crecimiento medio de 5\% en el caso de los AML solitarios).

Si bien aún no se cuenta con los resultados de un estudio prospectivo destinado a evaluar la evolución de AML de diversos tamaños en el largo plazo, la información obtenida hasta el momento permite establecer algunas pautas para el manejo de estas lesiones. En general los AML menores de $4 \mathrm{~cm} \mathrm{y}$ asintomáticos pueden ser seguidos con observación y evaluaciones repetidas cada 6 a 12 meses para determinar la velocidad de crecimiento y la importancia clínica de la lesión ${ }^{13}$. En el caso de tumores de mayor tamaño debe considerarse la posibilidad de una intervención quirúrgica, sobre todo si el paciente es sintomático, teniendo presentes la edad, patologías coexistentes y otros factores relacionados. En el caso de pacientes con AML pequeños que requieren intervención debido a la presencia de síntomas, en pacientes con ET o AML multicéntricos y en pacientes en quienes la preservación de la función renal reviste particular importancia el enfoque preferido es la cirugía ahorradora de nefronas con nefrectomía parcial o embolización selectiva ${ }^{11-13}$.

La mayoría de los pacientes con una hemorragia aguda o potencialmente fatal requieren una nefrectomía total, y en pacientes con ET, enfermedad bilateral, insuficiencia renal previa $\mathrm{u}$ otros trastornos con riesgo de deterioro funcional renal en el largo plazo, debe considerarse la embolización selectiva ${ }^{13}$. 


\section{REFERENCIAS}

1. Verdu Tartajo F, Herranz F, Subirá Rios D. Angiomiolipoma renal. Tumores del Riñón. 2001;241-245.

2. Wunderlich C R A: Handbuch der Pathologie und Therapie, 2nd ed. Stuttgart: Ebner andSeubert, 1856.

3. Regueiro Lopez JC, Molina Sanchez J, Carazo Carazo JL. Angiomiolipoma renal. Neoplasias renales. Diagnóstico y tratamiento. 2001; 43-56.

4. Algaba F, Solle Balcells FJ. Angiomiolipoma renal con extensión a ganglios. Act. Fund. Puigvert 1987; 6: 55-58.

5. Blute ML, Malek RS, Segura JW: Angiomyolipoma: Clinical metamorphosis and concepts for management. J Urol 1988;139: 20-24.

6. Bosniak MA, Megibow AJ, Hulnick DH, et al: CT diagnosis of renal angiomyolipoma: The importance of detecting small amounts of fat. AJR Am J Roentgenol 1998;151:497-501.

7. Bernstein J, Robbins O, Kissane JM: The renal lesions of tuberous sclerosis. Semin Diagn.Pathol. 1986;3:97-105

8. Antonpoulos P, Drossos C H, Triantopoulou C et al. Complications of renal angiomyolipomas: CT evaluation. Abdom Imaging 1996;21:357.
9. Burgos FJ, Mayayo T, Berenguer A, Maganto E, Mateos A Escudero A, Romero-Aguirre C. Angiomiolipoma renal. Aspectos diagnósticos y terapéuticos. Act Urol Esp.1988;12(3):210-216.

10. Escudero A, Martinez Piñeiro JA. Angiomiolipoma renal. Estudio de 12 casos. Arch. Esp Urol.1975;29:4-8.

11. Jardin A, Richard F, Le Duc A, Chatelain C, Le Guillou M Fourcade R, et al. Diagnosis and treatment of renal angiomyolipoma (based on 15 cases). Arguments in favor of conservative surgery (based on 8 cases). Eur Urol. 1980;6(2):69-82.

12. Zurera Tendero LJ,Delgado F, Ysamat Marfa R. Hemorragia retroperitoneal como complicación del angiomiolipoma renal. Arch Esp Urol. 1990;43(1):27-31.

13. Zhang JQ, Fielding JR, Zou KH. Etiology of spontaneous perirrenal hemorrhage: a meta-analysis. J Urol. 2002;167(4):1593-1596.

Correspondencia autor: Dr. J G Pedemonte

Servicio de urología. Hospital Italiano Garibaldi.

Virasoro 1249 - 2000. Rosario. Santa Fe. Argentina

E-mail autor: jgpedemonte@yahoo.com.ar

Información artículo: Nota Clínica

Trabajo recibido: febrero 2007

Trabajo aceptado: marzo 2007 